\title{
Oscillations of delay difference equations
}

\author{
X.H. TANG and J.S. YU \\ (Received April 16, 1999)
}

\begin{abstract}
We obtain some new sufficient conditions for oscillations of all solutions of the delay difference equation

$$
y_{n+1}-y_{n}+p_{n} y_{n-k}=0, \quad n=0,1,2, \ldots
$$

where $\left\{p_{n}\right\}$ is a sequence of nonnegative numbers and $k$ is a positive integer. Our theorems improve several previous well-known results. Some examples are given to demonstrate the advantage of our results.
\end{abstract}

Key words: oscillation, eventually positive solution, difference equation.

\section{Introduction}

In the recent papers [1-12], the oscillation of all solutions of the delay difference equation

$$
y_{n+1}-y_{n}+p_{n} y_{n-k}=0, \quad n=0,1,2, \ldots
$$

has been investigated, where $\left\{p_{n}\right\}$ is a sequence of nonnegative numbers and $k$ is a positive integer.

A solution $\left\{y_{n}\right\}$ of Eq.(1) is said to be oscillatory if the terms $y_{n}$ of the sequence are not eventually positive or eventually negative. Otherwise, the solution is called nonoscillatory.

In [1], Erbe and Zhang first proved that all solutions of (1) oscillate if

$$
\liminf _{n \rightarrow \infty} p_{n}>\frac{k^{k}}{(k+1)^{k+1}}
$$

or

$$
\Lambda=\limsup _{n \rightarrow \infty} \sum_{i=n-k}^{n} p_{i}>1 .
$$

1991 Mathematics Subject Classification : 39A10.

This work was supported by the grant for Ph.D. of National Educational Committee of China. 
Later, condition (2) was improved, by Ladas, Philos, Sficas [2], to

$$
\alpha=\liminf _{n \rightarrow \infty} \sum_{i=n-k}^{n-1} p_{i}>\left(\frac{k}{k+1}\right)^{k+1} .
$$

We remark that conditions (3) and (4) are two well-known oscillation criterion for (1) which have been extensively employed in the study of oscillation of various delay differences. For example, see the monographs $[3,4,5]$. However, there is an obvious gap between the conditions (3) and (4). It would be interesting to fill the gap, i.e. to obtain sufficient conditions for the oscillation of (1) when $\alpha \leq k^{k+1} /(k+1)^{k+1}$ and $\Lambda \leq 1$.

Recently, there are many papers which devoted oneself to filling the gap between conditions (3) and (4). For instance, Tang [6] proved that all solutions of (1) oscillate if

$$
\sum_{i=n-k}^{n-1} p_{i} \geq\left(\frac{k}{k+1}\right)^{k+1} \quad \text { for large } n
$$

and

$$
\sum_{n=k}^{\infty} p_{n}\left[\sum_{i=n-k}^{n-1} p_{i}-\left(\frac{k}{k+1}\right)^{k+1}\right]=\infty
$$

Clearly, conditions (5) and (6) improve (4). Afterwards, Tang and Yu [7] further improved the above conditions, proved that

$$
\sum_{n=0}^{\infty} p_{n}\left[(k+1)\left(\sum_{i=n+1}^{n+k} p_{i}\right)^{\frac{1}{k+1}}-k\right]=\infty
$$

also implies that all solutions of (1) oscillate.

In a different direction, Yu, Zhang and Qian [8] proved that all solutions of (1) oscillate if

$$
\alpha \leq\left(\frac{k}{k+1}\right)^{k+1} \quad \text { and } \quad \Lambda>1-\frac{\alpha^{2}}{4}
$$

or

$$
\alpha \leq\left(\frac{k}{k+1}\right)^{k+1} \quad \text { and } \quad \Lambda>\frac{2}{\sqrt{f(\alpha)}}
$$


where $f(\alpha) \in[1, k /(k+1) \alpha]$ satisfies the following equation

$$
f(\alpha)\left[1-\frac{\alpha}{k} f(\alpha)\right]^{k}=1 .
$$

In [9], Chen and Yu proved that (8) can be replaced by the weaker condition

$$
\alpha \leq\left(\frac{k}{k+1}\right)^{k+1} \quad \text { and } \quad \Lambda>1-\frac{1-\alpha-\sqrt{1-2 \alpha-\alpha^{2}}}{2} .
$$

Conditions (8), (9) and (11) all improve (3), but (8) and (11) are independent of (9).

The aim in this note is to further improve conditions (9) and (11). As a consequent of our main results, we prove that

$$
\alpha \leq\left(\frac{k}{k+1}\right)^{k+1} \text { and } \Lambda>\frac{1+\ln f(\alpha)}{f(\alpha)}-\frac{1-\alpha-\sqrt{1-2 \alpha-\alpha^{2}}}{2}
$$

guarantee that all solutions of (1) oscillate, where $f(\alpha)$ is the value determined by $\alpha$ from (10). It is not difficult to verify that (12) improves (9) and (11).

\section{Preliminaries}

For $0<\alpha \leq k^{k+1} /(k+1)^{k+1}$, since the function $x(1-\alpha x / k)^{k}$ is strictly increasing in $[1, k /(k+1) \alpha]$ from $(1-\alpha / k)^{k}$ to $(1 / \alpha) k^{k+1} /(k+1)^{k+1}$, it follows there exists a unique function $f(\alpha) \in[1, k /(k+1) \alpha]$ such that (10) holds. It is easy to see that $f(0)=1, f\left(k^{k+1} /(k+1)^{k+1}\right)=(k+1)^{k} / k^{k}$, and $1<f(\alpha)<k /(k+1) \alpha$ for $0<\alpha<k^{k+1} /(k+1)^{k+1}$. From this and (10)] we obtain

$$
\left[1-\frac{k+1}{k} \alpha f(\alpha)\right] f^{\prime}(\alpha)=f^{2}(\alpha)
$$

which leads to that $f^{\prime}(\alpha)>0$ for $0<\alpha<k^{k+1} /(k+1)^{k+1}$. This shows that function $f(\alpha)$ is strictly increasing in $\left[0, k^{k+1} /(k+1)^{k+1}\right]$.

Lemma 1 [9] Assume that $0 \leq \alpha \leq k^{k+1} /(k+1)^{k+1}$, and let $\left\{y_{n}\right\}$ be an 
eventually positive solution of (1). Then

$$
\liminf _{n \rightarrow \infty} \frac{y_{n+1}}{y_{n-k}} \geq A(\alpha): \frac{1-\alpha-\sqrt{1-2 \alpha-\alpha^{2}}}{2} .
$$

Lemma 2 [8] Assume that $0 \leq \alpha \leq k^{k+1} /(k+1)^{k+1}$, and let $\left\{y_{n}\right\}$ be an eventually positive solution of (1). Then

$$
\liminf _{n \rightarrow \infty} \frac{y_{n-k}}{y_{n}} \geq f(\alpha)
$$

Lemma 3 Assume that $0 \leq \alpha \leq k^{k+1} /(k+1)^{k+1}$, and let $\left\{y_{n}\right\}$ be an eventually positive solution of (1). Then

$$
\limsup _{n \rightarrow \infty} p_{n} \leq \frac{1}{f(\alpha)}-A(\alpha)
$$

Proof. From (1), we have eventually

$$
p_{n}=\frac{y_{n}}{y_{n-k}}-\frac{y_{n+1}}{y_{n-k}} \text {. }
$$

By Lemmas 1 and 2, it follows from (16) that

$$
\limsup _{n \rightarrow \infty} p_{n} \leq 1 / \liminf _{n \rightarrow \infty} \frac{y_{n-k}}{y_{n}}-\liminf _{n \rightarrow \infty} \frac{y_{n+1}}{y_{n-k}} \leq \frac{1}{f(\alpha)}-A(\alpha) .
$$

The proof is complete.

Lemma 4 Assume that $0 \leq \alpha \leq k^{k+1} /(k+1)^{k+1}$, and let $\left\{y_{n}\right\}$ be an eventually positive solution of (1). Then

$$
\liminf _{n \rightarrow \infty}\left[\frac{y_{n-k}}{y_{n}} \prod_{i=n-k}^{n-1}\left(1-p_{i} f(\alpha)\right)\right] \geq 1 .
$$

Proof. Let $n_{0}>0$ be an integer such that $y_{n}>0$ and $y_{n+1}-y_{n} \leq 0$ for $n \geq n_{0}-2 k$. From (1), we have

$$
\frac{y_{n-k}}{y_{n}}=\prod_{i=n-k}^{n-1}\left(1-p_{i} \frac{y_{i-k}}{y_{i}}\right)^{-1}, \quad n \geq n_{0} .
$$


If $\alpha=0$, then $f(\alpha)=1$. It follows from (18) that

$$
\frac{y_{n-k}}{y_{n}} \geq \prod_{i=n-k}^{n-1}\left(1-p_{i}\right)^{-1}, \quad n \geq n_{0}
$$

or

$$
\frac{y_{n-k}}{y_{n}} \prod_{i=n-k}^{n-1}\left(1-p_{i}\right) \geq 1, \quad n \geq n_{0}
$$

which implies that (17) holds for the case $\alpha=0$. If $0<\alpha \leq k^{k+1} /(k+1)^{k+1}$, then $f(\alpha)>1$ and $A(\alpha)>0$. By Lemma 3, there exists an integer $n_{1}>$ $n_{0}+k$ such that

$$
p_{n} \leq \frac{1}{f(\alpha)}-\frac{1}{2} A(\alpha) \quad \text { for } \quad n \geq n_{1} .
$$

Set $\omega_{n}=\min \left\{y_{n-k} / y_{n}, f(\alpha)\right\}$ for $n \geq n_{0}$. Then by Lemma 2, $\liminf _{n \rightarrow \infty} \omega_{n}=f(\alpha)$. Hence, from (18), we obtain

$$
\frac{y_{n-k}}{y_{n}} \geq \prod_{i=n-k}^{n-1}\left(1-p_{i} \omega_{i}\right)^{-1}, \quad n \geq n_{1} .
$$

It follows that for $n \geq n_{1}+k$

$$
\begin{aligned}
\frac{y_{n-k}}{y_{n}} \prod_{i=n-k}^{n-1}\left(1-p_{i} f(\alpha)\right) & \geq \prod_{i=n-k}^{n-1} \frac{1-p_{i} f(\alpha)}{1-p_{i} \omega_{i}} \\
& \geq \prod_{i=n-k}^{n-1} \frac{1-\left[\frac{1}{f(\alpha)}-\frac{1}{2} A(\alpha)\right] f(\alpha)}{1-\left[\frac{1}{f(\alpha)}-\frac{1}{2} A(\alpha)\right] \omega_{i}} \\
& =\prod_{i=n-k}^{n-1} \frac{\frac{1}{2} A(\alpha) f(\alpha)}{1-\left[\frac{1}{f(\alpha)}-\frac{1}{2} A(\alpha)\right] \omega_{i}}
\end{aligned}
$$

and so

$$
\begin{aligned}
\liminf _{n \rightarrow \infty}\left[\frac{y_{n-k}}{y_{n}} \prod_{i=n-k}^{n-1}\left(1-p_{i} f(\alpha)\right)\right] & \geq\left[\frac{\frac{1}{2} A(\alpha) f(\alpha)}{1-\left[\frac{1}{f(\alpha)}-\frac{1}{2} A(\alpha)\right] \liminf _{n \rightarrow \infty} \omega_{n}}\right]^{k} \\
& =1 .
\end{aligned}
$$

The proof is complete. 


\section{Main Results}

The first Theorem is a direct corollary of Lemma 3.

Theorem 1 Assume that $0 \leq \alpha \leq k^{k+1} /(k+1)^{k+1}$. If

$$
\limsup _{n \rightarrow \infty} p_{n}>\frac{1}{f(\alpha)}-A(\alpha)
$$

then all solutions of (1) oscillate.

Next we are going to deal with the case when the inequality in condition (20) is reversed. Without loss of generality, we assume that

$$
p_{n} \leq \frac{1}{f(\alpha)}-\frac{1}{2} A(\alpha) \text { for } n=0,1,2, \ldots .
$$

Theorem 2 Assume that $0 \leq \alpha \leq k^{k+1} /(k+1)^{k+1}$. If

$$
\limsup _{n \rightarrow \infty} \sum_{i=n-k}^{n} p_{i} \prod_{j=i-k}^{n-k-1}\left(1-p_{j} f(\alpha)\right)^{-1}>1-\frac{1-\alpha-\sqrt{1-2 \alpha-\alpha^{2}}}{2}
$$

then all solutions of (1) oscillate.

Proof. Suppose the contrary,and let $\left\{y_{n}\right\}$ be an eventually positive solution of (1). Then there exists an integer $n_{0}>k$ such that

$$
y_{n}>0 \quad \text { and } \quad y_{n+1}-y_{n} \leq 0, \quad n \geq n_{0}-k .
$$

For the case $\alpha=0$, since $f(\alpha)=1,(21)$ reduces to

$$
\limsup _{n \rightarrow \infty} \sum_{i=n-k}^{n} p_{i} \prod_{j=i-k}^{n-k-1}\left(1-p_{j}\right)^{-1}>1
$$

From (1), we have

$$
\frac{y_{n+1}}{y_{n}}=1-p_{n} \frac{y_{n-k}}{y_{n}}, \quad n \geq n_{0} .
$$

It follows that for $n-k \leq i \leq n$ and $n \geq n_{0}+k$

$$
\frac{y_{i-k}}{y_{n-k}}=\prod_{j=i-k}^{n-k-1}\left(1-p_{j} \frac{y_{j-k}}{y_{j}}\right)^{-1} \text {. }
$$


Note that $y_{n-k} / y_{n} \geq 1$ for $n \geq n_{0}$, from (23) we get

$$
\frac{y_{i-k}}{y_{n-k}} \geq \prod_{j=i-k}^{n-k-1}\left(1-p_{j}\right)^{-1}, \quad n \geq n_{0}+k \quad \text { and } \quad n-k \leq i \leq n .
$$

Summing (1) from $n-k$ to $n$ and using the above inequalities, we obtain

$$
\begin{aligned}
y_{n-k}-y_{n+1} & =\sum_{i=n-k}^{n} p_{i} y_{i-k} \\
& \geq y_{n-k} \sum_{i=n-k}^{n} p_{i} \prod_{j=i-k}^{n-k-1}\left(1-p_{j}\right)^{-1}, \quad n \geq n_{0}+k
\end{aligned}
$$

or

$$
1-\frac{y_{n+1}}{y_{n-k}} \geq \sum_{i=n-k}^{n} p_{i} \prod_{j=i-k}^{n-k-1}\left(1-p_{j}\right)^{-1}, \quad n \geq n_{0}+k
$$

Hence

$$
\limsup _{n \rightarrow \infty} \sum_{i=n-k}^{n} p_{i} \prod_{j=i-k}^{n-k-1}\left(1-p_{j}\right)^{-1} \leq 1
$$

which contradicts with $(22)$.

For the other case $0<\alpha \leq k^{k+1} /(k+1)^{k+1}$, we have $f(\alpha)>1$ and $A(\alpha)>0$. Rewrite $(21)$ as

$$
\limsup _{n \rightarrow \infty} \sum_{i=n-k}^{n} p_{i} \prod_{j=i-k}^{n-k-1}\left(1-p_{j} f(\alpha)\right)^{-1}+A(\alpha)>1
$$

This implies that there exists $\eta \in(1 / f(\alpha), 1)$ such that

$$
\limsup _{n \rightarrow \infty} \lambda^{k} \sum_{i=n-k}^{n} p_{i} \prod_{j=i-k}^{n-k-1}\left(1-p_{j} f(\alpha)\right)^{-1}+\eta A(\alpha)>1 .
$$

where

$$
\lambda=\frac{f(\alpha) A(\alpha)}{2(1-\eta)+\eta f(\alpha) A(\alpha)} .
$$


By Lemmas 1 and 2, there exists an integer $n_{1}>n_{0}$ such that

$$
\frac{y_{n-k}}{y_{n}} \geq \eta f(\alpha) \quad \text { and } \quad \frac{y_{n+1}}{y_{n-k}} \geq \eta A(\alpha), \quad n \geq n_{1} .
$$

From (25), we may choose an integer $N>n_{1}+2 k$ so large that

$$
\lambda^{k} \sum_{i=N-k}^{N} p_{i} \prod_{j=i-k}^{N-k-1}\left(1-p_{j} f(\alpha)\right)^{-1}+\eta A(\alpha)>1 .
$$

On the other hand, from (23), (26) and (27), we have for $N-k \leq i \leq N$

$$
\begin{aligned}
\frac{y_{i-k}}{y_{N-k}} & =\prod_{j=i-k}^{N-k-1}\left(1-p_{j} \frac{y_{j-k}}{y_{j}}\right)^{-1} \geq \prod_{j=i-k}^{N-k-1}\left(1-p_{j} \eta f(\alpha)\right)^{-1} \\
& =\prod_{j=i-k}^{N-k-1} \frac{\lambda}{\lambda-1+p_{j} f(\alpha)(1-\lambda \eta)+1-p_{j} f(\alpha)} \\
& \geq \prod_{j=i-k}^{N-k-1} \frac{\lambda}{\lambda-1+[1-f(\alpha) A(\alpha) / 2](1-\lambda \eta)+1-p_{j} f(\alpha)} \\
& =\prod_{j=i-k}^{N-k-1} \lambda\left(1-p_{j} f(\alpha)\right)^{-1} \geq \lambda^{k} \prod_{j=i-k}^{N-k-1}\left(1-p_{j} f(\alpha)\right)^{-1} .
\end{aligned}
$$

Summing (1) from $N-k$ to $N$ and using the above inequalities, we obtain

$$
\begin{aligned}
y_{N-k}-y_{N+1} & =\sum_{i=N-k}^{N} p_{i} y_{i-k} \\
& \geq \lambda^{k} y_{N-k} \sum_{i=N-k}^{N} p_{i} \prod_{j=i-k}^{N-k-1}\left(1-p_{j} f(\alpha)\right)^{-1}
\end{aligned}
$$

or

$$
1-\frac{y_{N+1}}{y_{N-k}} \geq \lambda^{k} \sum_{i=N-k}^{N} p_{i} \prod_{j=i-k}^{N-k-1}\left(1-p_{j} f(\alpha)\right)^{-1} .
$$

Substituting $y_{N+1} / y_{N-k} \geq \eta A(\alpha)$ into (29), we have

$$
1 \geq \eta A(\alpha)+\lambda^{k} \sum_{i=N-k}^{N} p_{i} \prod_{j=i-k}^{N-k-1}\left(1-p_{j} f(\alpha)\right)^{-1}
$$


which contradicts with (28), and so the proof is complete.

Theorem 3 Assume that $0 \leq \alpha \leq k^{k+1} /(k+1)^{k+1}$. If

$$
\begin{gathered}
\limsup _{n \rightarrow \infty} \sum_{i=n-k}^{n} p_{i}\left[\min \left\{\prod_{j=i-k}^{n-k-1}\left(1-p_{j} f(\alpha)\right)^{-1}, \frac{1}{f(\alpha)} \prod_{j=i-k}^{i-1}\left(1-p_{j} f(\alpha)\right)^{-1}\right\}\right] \\
>\frac{1+\ln f(\alpha)}{f(\alpha)}-\frac{1-\alpha-\sqrt{1-2 \alpha-\alpha^{2}}}{2}
\end{gathered}
$$

then all solutions of (1) oscillate.

Proof. For the case $\alpha=0$, since $f(\alpha)=1$, it is easy to see that (30) is the same to (21). By Theorem 2, the conclusion of Theorem 3 is true. In the sequel, we only consider the other case $0<\alpha \leq k^{k+1} /(k+1)^{k+1}$. Suppose that the conclusion of the theorem is false, and that (1) has an eventually positive solution $\left\{y_{n}\right\}$. Choose a positive integer $n_{0}>k$ such that $y_{n}>0$ and $y_{n+1}-y_{n} \leq 0$ for $n \geq n_{0}-k$. Rewrite (30) as

$$
\begin{gathered}
\limsup _{n \rightarrow \infty} \sum_{i=n-k}^{n} p_{i}\left[\min \left\{\prod_{j=i-k}^{n-k-1}\left(1-p_{j} f(\alpha)\right)^{-1}, \frac{1}{f(\alpha)} \prod_{j=i-k}^{i-1}\left(1-p_{j} f(\alpha)\right)^{-1}\right\}\right] \\
+A(\alpha)-\frac{1+\ln f(\alpha)}{f(\alpha)}>0
\end{gathered}
$$

Since $f(\alpha)>1$ and $A(\alpha)>0,(31)$ implies that there exists $\eta \in(1 / f(\alpha), 1)$ such that

$$
\begin{gathered}
\limsup _{n \rightarrow \infty} \lambda^{k} \sum_{i=n-k}^{n} p_{i}\left[\min \left\{\prod_{j=i-k}^{n-k-1}\left(1-p_{j} f(\alpha)\right)^{-1}, \frac{1}{f(\alpha)} \prod_{j=i-k}^{i-1}\left(1-p_{j} f(\alpha)\right)^{-1}\right\}\right] \\
+\eta A(\alpha)-\frac{1+\ln \eta f(\alpha)}{\eta f(\alpha)}>0
\end{gathered}
$$

where $\lambda$ is defined by (26). For given $\eta$, by Lemmas 1,2 and 4, there exists an integer $n_{1}>n_{0}+k$ such that for $n \geq n_{1}$

$$
\frac{y_{n-k}}{y_{n}} \geq \eta f(\alpha) \quad \text { and } \quad \frac{y_{n+1}}{y_{n-k}} \geq \eta A(\alpha)
$$


and

$$
\frac{y_{n-k}}{y_{n}} \prod_{j=n-k}^{n-1}\left(1-p_{j} f(\alpha)\right) \geq \eta .
$$

It follows from (32) that there exists an integer $N>n_{1}+2 k$ such that

$$
\begin{gathered}
\lambda^{k} \sum_{i=N-k}^{N} p_{i}\left[\min \left\{\prod_{j=i-k}^{N-k-1}\left(1-p_{j} f(\alpha)\right)^{-1}, \frac{1}{f(\alpha)} \prod_{j=i-k}^{i-1}\left(1-p_{j} f(\alpha)\right)^{-1}\right\}\right] \\
>\frac{1+\ln \eta f(\alpha)}{\eta f(\alpha)}-\eta A(\alpha) .
\end{gathered}
$$

Since

$$
y_{N-k} / y_{N-k}=1 \quad \text { and } \quad y_{N-k} / y_{N}>\eta f(\alpha)>1 .
$$

Then there exists an integer $l$ with $0 \leq l \leq k$ such that

$$
y_{N-k} / y_{N-l} \leq \eta f(\alpha) \text { and } y_{N-k} / y_{N-l+1}>\eta f(\alpha) .
$$

Let $\xi \in[0,1)$ such that

$$
y_{N-k} /\left[y_{N-l}+\xi\left(y_{N-l+1}-y_{N-l}\right)\right]=\eta f(\alpha) .
$$

From (1) and (34), we have for $t \in[0,1]$ and $n \geq n_{0}+k$

$$
\begin{aligned}
-\frac{y_{n+1}-y_{n}}{y_{n}+t\left(y_{n+1}-y_{n}\right)} & =p_{n} \frac{y_{n-k}}{y_{n}+t\left(y_{n+1}-y_{n}\right)} \geq p_{n} \frac{y_{n-k}}{y_{n}} \\
& \geq \eta p_{n} \prod_{j=n-k}^{n-1}\left(1-p_{j} f(\alpha)\right)^{-1} .
\end{aligned}
$$

For $n \in\{N-k, N-k+1, \ldots, N-l-1\}$, integrating (37)] over [0,1], we get

$$
\begin{aligned}
\ln \frac{y_{n}}{y_{n+1}} \geq \eta p_{n} \prod_{j=n-k}^{n-1}\left(1-p_{j} f(\alpha)\right)^{-1} & \\
& n=N-k, N-k+1, \ldots, N-l-1 .
\end{aligned}
$$

For $n=N-l$, integrating again (37) over $[0, \xi]$, we have

$$
\ln \frac{y_{N-l}}{y_{N-l}+\xi\left(y_{N-l+1}-y_{N-l}\right)} \geq \xi \eta p_{N-l} \prod_{j=N-k-l}^{N-l-1}\left(1-p_{j} f(\alpha)\right)^{-1} \text {. }
$$


Summing the above inequalities, we obtain

$$
\begin{aligned}
\ln \frac{y_{N-k}}{y_{N-l}+\xi\left(y_{N-l+1}-y_{N-l}\right)} \geq & \eta \sum_{i=N-k}^{N-l-1} p_{i} \prod_{j=i-k}^{i-1}\left(1-p_{j} f(\alpha)\right)^{-1} \\
& +\xi \eta p_{N-l} \prod_{j=N-k-l}^{N-l-1}\left(1-p_{j} f(\alpha)\right)^{-1} .
\end{aligned}
$$

In view of (36),

$$
\begin{aligned}
\frac{\ln \eta f(\alpha)}{\eta f(\alpha)} \geq \frac{1}{f(\alpha)}\left[\sum_{i=N-k}^{N-l-1} p_{i} \prod_{j=i-k}^{i-1}\left(1-p_{j} f(\alpha)\right)^{-1}\right. \\
\\
\left.\quad+\xi p_{N-l} \prod_{j=N-k-l}^{N-l-1}\left(1-p_{j} f(\alpha)\right)^{-1}\right] .
\end{aligned}
$$

Similar to proof Theorem 2, from (1), (26) and (33) we may obtain

$$
\begin{aligned}
\frac{y_{i-k}}{y_{N-k}} & =\prod_{j=i-k}^{N-k-1}\left(1-p_{j} \frac{y_{j-k}}{y_{j}}\right)^{-1} \\
& \geq \lambda^{k} \prod_{j=i-k}^{N-k-1}\left(1-p_{j} f(\alpha)\right)^{-1}, \quad N-k \leq i \leq N .
\end{aligned}
$$

Hence

$$
\begin{aligned}
& y_{N-l}+\xi\left(y_{N-l+1}-y_{N-l}\right)-y_{N+1} \\
&=-\sum_{i=N-l}^{N}\left(y_{i+1}-y_{i}\right)+\xi\left(y_{N-l+1}-y_{N-l}\right) \\
&= \sum_{i=N-l+1}^{N} p_{i} y_{i-k}+(1-\xi) p_{N-l} y_{N-l-k} \\
& \geq \lambda^{k} y_{N-k}\left[\sum_{i=N-l+1}^{N} p_{i} \prod_{j=i-k}^{N-k-1}\left(1-p_{j} f(\alpha)\right)^{-1}\right. \\
&\left.\quad+(1-\xi) p_{N-l} \prod_{j=N-k-l}^{N-k-1}\left(1-p_{j} f(\alpha)\right)^{-1}\right]
\end{aligned}
$$


It follows that

$$
\begin{aligned}
& \frac{y_{N-l}+\xi\left(y_{N-l+1}-y_{N-l}\right)}{y_{N-k}}-\frac{y_{N+1}}{y_{N-k}} \\
& \geq \lambda^{k}\left[\sum_{i=N-l+1}^{N} p_{i} \prod_{j=i-k}^{N-k-1}\left(1-p_{j} f(\alpha)\right)^{-1}\right. \\
& \left.\quad+(1-\xi) p_{N-l} \prod_{j=N-k-l}^{N-k-1}\left(1-p_{j} f(\alpha)\right)^{-1}\right]
\end{aligned}
$$

Substituting (33) and (36) into this,

$$
\begin{aligned}
& \frac{1}{\eta f(\alpha)}- \eta A(\alpha) \\
& \geq \lambda^{k}\left[\sum_{i=N-l+1}^{N} p_{i} \prod_{j=i-k}^{N-k-1}\left(1-p_{j} f(\alpha)\right)^{-1}\right. \\
&\left.+(1-\xi) p_{N-l} \prod_{j=N-k-l}^{N-k-1}\left(1-p_{j} f(\alpha)\right)^{-1}\right]
\end{aligned}
$$

Adding (38) and (39) leads to

$$
\begin{aligned}
& \frac{1+\ln \eta f(\alpha)}{\eta f(\alpha)}-\eta A(\alpha) \\
& \geq \lambda^{k} \sum_{i=N-k}^{N} p_{i}\left[\min \left\{\prod_{j=i-k}^{N-k-1}\left(1-p_{j} f(\alpha)\right)^{-1}, \frac{1}{f(\alpha)} \prod_{j=i-k}^{i-1}\left(1-p_{j} f(\alpha)\right)^{-1}\right\}\right]
\end{aligned}
$$

which contradicts with (35), and so the proof is complete.

From Theorems 2 and 3, we have immediately

Corollary 1 Assume that $0 \leq \alpha \leq k^{k+1} /(k+1)^{k+1}$. If

$$
\limsup _{n \rightarrow \infty} \sum_{i=n-k}^{n} p_{i} \prod_{j=i-k}^{n-k-1}\left(1-p_{j}\right)^{-1}>1-\frac{1-\alpha-\sqrt{1-2 \alpha-\alpha^{2}}}{2}
$$

then all solutions of (1) oscillate. 
Corollary 2 Assume that $0 \leq \alpha \leq k^{k+1} /(k+1)^{k+1}$. If

$$
\Lambda=\limsup _{n \rightarrow \infty} \sum_{i=n-k}^{n} p_{i}>\frac{1+\ln f(\alpha)}{f(\alpha)}-\frac{1-\alpha-\sqrt{1-2 \alpha-\alpha^{2}}}{2},
$$

then all solutions of (1) oscillate.

Remark 1. Obviously, Condition (41) improves (9) and (11) when $0 \leq$ $\alpha \leq k^{k+1} /(k+1)^{k+1}$. However, as $\alpha \rightarrow 0,(41)$, together with (8), (9) and (11), reduces to (3). Nevertheless, the following Example 2 illustrate that Corollary 1 still possible improve (3) for the case $\alpha=0$.

\section{Several Examples}

In this section, we give some examples to show the effect of our results. Example 1. Consider the difference equation

$$
y_{n+1}-y_{n}+p_{n} y_{n-2}=0, \quad n=1,2, \ldots,
$$

where $p_{10 n}=p_{10 n+1}=\cdots=p_{10 n+8}=0.1, p_{10 n+9}=0.73, n=0,1,2, \ldots$ It is easy to observe that

$$
\begin{aligned}
& \alpha=\liminf _{n \rightarrow \infty} \sum_{i=n-2}^{n-1} p_{i}=0.2<\left(\frac{2}{3}\right)^{3}, \\
& \Lambda=\limsup _{n \rightarrow \infty} \sum_{i=n-2}^{n} p_{i}=0.93<1 .
\end{aligned}
$$

In addition, we find

$$
f(\alpha)=1.336 \text { and } A(\alpha)=\left(1-\alpha-\sqrt{1-2 \alpha-\alpha^{2}}\right) / 2=0.026
$$

By these, one can easy verify that

$$
\Lambda<\frac{1+\ln f(\alpha)}{f(\alpha)}-A(\alpha) .
$$

These show conditions (3), (4), (8), (9), (11) and (12) are not satisfied. But

$$
\limsup _{n \rightarrow \infty} p_{n}=0.73>\frac{1}{f(\alpha)}-A(\alpha)=0.7225
$$

Hence, the conditions of Theorem 1 are satisfied and therefore every solution 
of (42) is oscillatory.

Example 2. Consider the difference equation

$$
y_{n+1}-y_{n}+p_{n} y_{n-3}=0, \quad n=0,1,2, \ldots
$$

where $k=3$ and $p_{15 n}=p_{15 n+1}=\cdots=p_{15 n+7}=0, p_{15 n+8}=p_{15 n+9}=$ $\cdots=p_{15 n+14}=0.2, n=0,1,2, \ldots$ It is easy to observe that

$$
\begin{aligned}
& \alpha=\liminf _{n \rightarrow \infty} \sum_{i=n-3}^{n-1} p_{i}=0, \\
& \Lambda=\limsup _{n \rightarrow \infty} \sum_{i=n-3}^{n} p_{i}=0.8<1,
\end{aligned}
$$

which show that conditions (3), (4), (5), (9) and (11) are not satisfied. In addition, it is easy to verify that (7) is not satisfied either. But we

$$
\sum_{i=15 n+11}^{15 n+14} p_{i} \prod_{j=i-3}^{15 n+10}\left(1-p_{j}\right)^{-1}=\frac{369}{320}
$$

and so

$$
\limsup _{n \rightarrow \infty} \sum_{i=n-3}^{n} p_{i} \prod_{j=i-3}^{n-3-1}\left(1-p_{j}\right)^{-1}>1 .
$$

Hence, the conditions of Corollary 1 are satisfied and therefore all solution of (43) oscillate.

Example 3. Consider the difference equation

$$
y_{n+1}-y_{n}+p_{n} y_{n-3}=0, \quad n=1,2, \ldots,
$$

where $p_{15 n}=p_{15 n+1}=\cdots=p_{15 n+7}=0.1, p_{15 n+8}=p_{15 n+9}=\cdots=$ $p_{15 n+14}=0.16, n=0,1,2, \ldots$ It is easy to observe that

$$
\begin{aligned}
& \alpha=\liminf _{n \rightarrow \infty} \sum_{i=n-3}^{n-1} p_{i}=0.3<\left(\frac{3}{4}\right)^{4}, \\
& \Lambda=\limsup _{n \rightarrow \infty} \sum_{i=n-3}^{n-1} p_{i}=0.64<1, \\
& f(\alpha)=f(0.3)=1.842,
\end{aligned}
$$




$$
A(\alpha)=\left(1-\alpha-\sqrt{1-2 \alpha-\alpha^{2}}\right) / 2=0.0716
$$

Hence

$$
\frac{1+\ln f(\alpha)}{f(\alpha)}-A(\alpha)=0.802>\Lambda
$$

which shows that condition (41) is not satisfied. But

$$
\begin{aligned}
& \sum_{i=15 n+11}^{15 n+14} p_{i}\left[\min \left\{\prod_{j=i-3}^{15 n+10}\left(1-p_{j} f(\alpha)\right)^{-1}, \frac{1}{f(\alpha)} \prod_{j=i-3}^{i-1}\left(1-p_{j} f(\alpha)\right)^{-1}\right\}\right] \\
& =0.16 \sum_{i=15 n+11}^{15 n+14}\left[\operatorname { m i n } \left\{(1-0.16 \times 1.842)^{-(15 n+14-i)},\right.\right. \\
& \left.\left.\quad \frac{1}{1.842}(1-0.16 \times 1.842)^{-3}\right\}\right] \\
& =0.16\left(\frac{1.418^{3}}{1.842}+\frac{1.418^{3}}{1.842}+1.418+1\right)=0.882 \\
& >0.802=\frac{1+\ln f(\alpha)}{f(\alpha)}-A(\alpha) .
\end{aligned}
$$

These show that the conditions of Theorem 3 are satisfied and therefore every solution of (44) is oscillatory.

Remark 2. Example 3 shows that Theorem 3 can ameliorate Corollary 2 in general case.

\section{References}

[1] Erbe L.H. and Zhang B.G., Oscillations of discrete analogue of delay equations. Diff. Integral Equations 2, (1989), 300-309.

[2] Ladas G., Philos CH. and Sficas Y.G., Sharp conditions for the oscillations of delay difference equations. J. Appl. Math. Simulations 2, (1989), 101-112.

[3] Agarwal R.P., Difference Equations and Inequalities: Theory, Methods and Applications. Marcel Dekker, New York, 1992.

[4] Gyori I. and Ladas G., Oscillation Theory of Delay Differential Equations with Applications. Clarendon Press, Oxford, 1991.

[5] Kelley W.G. and Peterson A.C., Difference Equations: An Introduction with Applications. Academic Press, New York, 1991.

[6] Tang X.H., Oscillation of delay difference equations with variable coefficients. J. Central South University of Technology, 29(4), (1998), 489-492 (Chinese).

[7] Tang X.H. and Yu J.S., Oscillation of delay difference equations. Computer Math. Appl. 37 (1999), 11-20. 
[8] Yu J.S., Zhang B.G. and Qian X.Z., Oscillations of delay difference equations with oscillating coefficients. J. Math. Anal. Appl. 177, (1993), 432-444.

[9] Chen Ming-Po and Yu J.S., Oscillations for delay difference equations with variable coefficients. Proceedings of the first International Conference on Difference Equations, Edited by Saber N.Elaydi, etc., Gordon and Breach Publishers 1994, 105-114.

[10] Ladas G., Explicit conditions for the oscillation of difference equations. J. Math. Anal. Appl. 153, (1990), 276-287.

[11] Philos CH., Oscillations of some difference equations. Funk. Ekvac. 34, (1991), 157172.

[12] Ladas G., Recent developments in the oscillation of delay difference equations, "Differential Equations: Stability and Control". Lectures notes in pure and applied mathematics, Vol.127, Marcel Dekker, New York (1991), 321-332.

X.H. Tang

Department of Applied Mathematics

Central South University of Technology

Changsha, Hunan 410083

P.R. China

J.S. Yu

Department of Applied Mathematics

Hunan University

Changsha, Hunan 410082

P.R. China

E-mail: jsyu@mail.hunu.edu.cn 